\title{
NOUVELLE
}

\section{Neuropathies périphériques et petites protéines de choc thermique}

\author{
Anne Tarrade, Coralie Fassier, Judith Melki
}

\author{
Laboratoire de \\ Neurogénétique moléculaire, \\ Inserm $\varepsilon$-0223, \\ Université d'Évry. \\ 2, rue Gaston Crémieux, \\ CP 5724,
}

91057 Évry Cedex, France. j.melki@genopole.inserm.fr famille pour laquelle le diagnostic de CMT2F liée au chromosome 7q1l-q2l a été porté, l'équipe de $V$. Timmerman a pu, grâce à une analyse génétique, restreindre la région candidate à un intervalle de $10 \mathrm{cM}$ entre les marqueurs taires, dont la prévalence est d'environ $1 / 5000$, sont les maladies monogéniques du système nerveux les plus fréquentes. Ce grand groupe de maladies, très hétérogène tant sur le plan clinique que génétique, présente une caractéristique commune, l'atteinte progressive des nerfs périphériques. L'analyse des vitesses de conduction nerveuse des nerfs périphériques à l'aide de l'examen électromyographique permet de distinguer deux grands types de neuropathies, les formes démyélinisantes - incluant la maladie de Charcot-Marie-Tooth de type 1, CMTl - et les formes axonales, incluant les neuropathies motrices distales héréditaires (dHMN) et sensitivomotrices (CMT2) [1].

De nombreux gènes responsables de ces affections ont été identifiés. Ils codent pour des protéines impliquées dans des processus cellulaires très divers tels que la compaction de la gaine de myéline (PMP22, P0), l'assemblage des neurofilaments (NEFL), le transport axonal (KIFlB), le trafic intracellulaire (RAB7), l'apoptose (SPTLC1, LITAF), la transcription (EGR2), la traduction (GARS) et la transduction du signal (PRX, MTMR2 et 13) [2, 3]. Deux publications récentes, parues dans la revue Nature Genetics $[4,5]$, suggèrent l'émergence d'un nouveau mécanisme physiopathologique à partir de l'identification, par l'équipe de V. Timmerman, de deux gènes codant pour les petites protéines de choc thermique (sHSP), HSP22 et HSP27. Ces protéines appartiennent à la superfamille des sHSP connues pour être impliquées dans de nombreux processus cellulaires tels que l'aide au repliement des protéines, l'inhibition de l'apoptose, l'organisation du cytosquelette ou la suppression d'agrégats protéiques [6-10]. Ces travaux ont d'abord porté sur deux familles atteintes de $\mathrm{dHMN}$ de type II liées au chromosome 12q24.3. Cette axonopathie motrice pure est caractérisée par une atrophie et une paralysie des muscles distaux. Une analyse des haplotypes utilisant des marqueurs polymorphes a permis aux auteurs de restreindre la région génétique candidate de $5 \mathrm{Mb}$ à $1,7 \mathrm{Mb}$ entre les marqueurs D12S349 et PLA2G1B. Parmi les neuf gènes connus dans cet intervalle, cinq avaient été préalablement exclus et les quatre autres ont fait l'objet d'un séquençage systématique des exons et des jonctions exon-intron. Cette stratégie a permis d'identifier une mutation faux-sens dans l'exon 2 du gène HSPB8 codant pour HSP22. Par la suite, une autre mutation faux-sens a été identifiée chez deux autres familles. Ces deux mutations conduisent à la substitution du résidu lysine en position 141 par un résidu asparagine (K141N) ou un acide glutamique (K14lع).

Cette équipe s'est également intéressée aux CMT2. Cette neuropathie sensitivomotrice à prédominance distale se caractérise par une faiblesse musculaire et une atrophie des muscles distaux associées à des anomalies sensitives distales. Cette axonopathie est génétiquement très hétérogène. Par exemple, dans les formes autosomiques dominantes, huit locus ont été identifiés et cinq gènes sont connus à ce jour (KIFIB, RAB7, GARS, NEFL, MPZ) [3]. Chez une
D7S672 et D7S806. Cinq gènes candidats ont été judicieusement sélectionnés dans cette région et, là encore, le séquençage systématique de ces gènes a permis d'identifier une mutation dans l'un d'entre eux. De façon surprenante, cette mutation faux-sens affecte le gène HSPBI codant lui aussi pour une sHSP, HSP27. La transition 404C->T identifiée dans ce gène conduit à la substitution d'un résidu sérine par un résidu phénylalanine en position 135 (S135F).

L'identification de mutations de HSP22 dans les dHMN, de mutations de HSP27 dans les CMT2, et le fait que ces deux protéines interagissent, ont conduit à rechercher des mutations de HSP27 dans d'autres familles atteintes de dHMN ou de CMT. Ce même groupe a ainsi identifié des mutations faux-sens de HSP27 chez des familles atteintes de dHMN, démontrant que les mutations de ce gène pouvaient conduire à des neuropathies purement motrices (dHMN) ou à des neuropathies sensitivo-motrices (CMT2). L'ensemble de ces résultats suggère donc l'implication des sHSP dans les neuropathies périphériques.

Plusieurs arguments permettent de démontrer la responsabilité des mutations faux-sens de ces gènes dans la pathogénie de ces affections. HSP22 et HSP27 sont avant tout des protéines ubiquitaires, bien exprimées dans les neurones sensitifs et moteurs affectés dans ces neuropathies. Ces mutations n'ont pas été retrouvées au sein d'une grande population de sujets sains, un argument 
faible pour des maladies rares. De plus, les mutations observées chez ces patients sont localisées dans le domaine $\alpha$-cristalline, fortement conservé au sein de la famille des protéines SHSP, ou dans d'autres résidus très conservés au sein des protéines orthologues. De plus, ces mutations sont situées à proximité de résidus d'autres sHSP telles que l' $\alpha-A$ cristalline et l' $\alpha$ - $B$ cristalline, dont les mutations faux-sens sont responsables respectivement d'une forme de cataracte congénitale ou d'une myopathie $[11,12]$. Le caractère «pathologique » de ces mutations a été confirmé en analysant leurs effets dans des modèles cellulaires. Une diminution de la survie des cellules de neuroblastomes exprimant l'une ou l'autre de ces protéines mutées (HSP22 K141E et HSP27 S135F) a souligné l'effet délétère des mutations de ces sHSP. De plus, des anomalies intracellulaires ont été observées. En effet, la mutation HSP22K14lE engendre la formation d'agrégats de HSP22 cytoplasmique et périnucléaire. Quant à la mutation HSP27S135F, observée dans les CMT2F, elle engendre in vitro un défaut d'assemblage des neurofilaments, rappelant celui déjà décrit dans les CMT2E liées aux mutations du gène NEFL. Des anomalies du cytosquelette telles que des agrégats de desmine ont également été observées dans une myopathie liée à des mutations du domaine $\alpha$-cristalline d'une autre sHSP, l' $\alpha-B$ cristalline [8]. L'altération des filaments intermédiaires suggère donc que le maintien de l'organisation du cytosquelette implique les sHSP. L'intégrité du cytosquelette étant indispensable au bon fonctionnement du transport axonal - un processus essentiel dans les neurones de très grande taille - il est possible que les mutations des sHSP conduisent à une altération de ce transport.

L'ensemble de ces travaux souligne plusieurs points. Le séquençage systématique des gènes situés dans un intervalle génétique important reste parfois le seul recours efficace pour permettre l'identification des mutations de gènes de mala- dies rares, quand le nombre de familles ne permet pas une cartographie génétique très fine. La génétique à rebours n'a donc pas fini de nous ouvrir de nouvelles portes vers des mécanismes physiopathologiques inexplorés! Cette stratégie a ainsi permis d'identifier des mutations au sein des gènes codant pour HSP22 et HSP27 dans les neuropathies périphériques. Ces travaux démontrent le rôle majeur des sHSP dans les maladies neurodégénératives. L'identification de ces deux gènes souligne de nouveau l'hétérogénéité génétique de ces affections. De plus, l'identification de mutations de HSP27 dans des neuropathies sensitivo-motrices, ou motrices pures, suggère l'implication de mécanismes physiopathologiques communs à l'origine de neuropathies pourtant cliniquement distinctes. Cette hypothèse est renforcée par les travaux récemment rapportés par A. Antonellis et al., qui montrent que des mutations du gène GARS codant pour la glycine ARNt synthétase sont responsables de neuropathies sensitivo-motrices (CMT2D) ou motrices pures (dHMN-v) [13]. Un nouvel exemple de l'absence de corrélation génotype-phénotype!

L'existence d'un rôle crucial des HSP dans les maladies neurodégénératives avait déjà été suspectée par l'identification d'une mutation de la protéine chaperon HSP60 au sein d'une famille atteinte de paraplégie spastique, une maladie caractérisée par une dégénérescence des axones des voies corticospinales [14]. De plus, HSP27 semble jouer un rôle neuroprotecteur dans certaines maladies [10, $15]$. Les protéines chaperons, et en particulier les petites protéines de choc thermique, jouent donc un rôle pivot dans l'intégrité du système nerveux. Néanmoins, les mécanismes moléculaires conduisant à la dégénérescence des axones plutôt que d'autres types cellulaires restent encore mal connus. De plus, la pathogénie des mutations de HSP27 ou HSP22 dans les neuropathies - effet dominant négatif ou gain d'une nouvelle fonction - reste à élucider.

Comment réconcilier cette extraordi- naire hétérogénéité génétique et les maladies du nerf périphérique que peu ou pas d'éléments cliniques permettent de distinguer? S'agit-il de processus physiopathologiques distincts d'un bout (la mutation génique) à l'autre (la dégénérescence axonale), ou de mécanismes physiopathologiques distincts qui s'engouffrent ensuite vers un même tronc commun pour aboutir à la dégénérescence axonale ? Dans le premier cas, cela conduit à une recherche physiopathologique et thérapeutique spécifique de chaque entité moléculaire. Dans le second cas, la dissection moléculaire de ce tronc commun pourrait conduire à l'identification de cibles thérapeutiques communes aux neuropathies périphériques. Une approche «transversale » de ces affections est désormais possible à partir de modèles murins ou cellulaires de ces maladies utilisant des techniques d'analyse globale du protéome ou du transcriptome. Elle devrait permettre de répondre à cette question clé pour les recherches thérapeutiques à venir. $\diamond$

Neuropathies and small heat shock proteins

\section{RÉFÉRENCES}

1. Kuhlenbaumer G, Young P, Hunermund G, et al. Clinical features and molecular genetics of hereditary peripheral neuropathies. J Neurol 2002; 249: 1629-50.

2. Klein CJ. Pathology and molecular genetics of inherited neuropathy.J Neurol Sci 2004; 220: 141-3.

3. Suter U, Scherer SS. Disease mechanisms in inherited neuropathies. Nat Rev Neurosci 2003; 4: 714-26.

4. Evgrafov OV, Mersiyanova I, Irobi J, et al. Mutant small heat-shock protein 27 causes axonal CharcotMarie-Tooth disease and distal hereditary motor neuropathy. Nat Genet 2004; 36: 602-6.

5. Irobi J, Van Impe K, Seeman P, et al. Hot-spot residue in small heat-shock protein 22 causes distal motor neuropathy. Nat Genet 2004; 36: 597-601.

6. Welsh MJ, Gaestel M. Small heat-shock protein family: function in health and disease. Ann NY Acad Sci 1998; 851: 28-35.

7. Bruey JM, Ducasse C, Bonniaud P, et al. Hsp27 negatively regulates cell death by interacting with cytochrome c. Nat Cell Biol 2000; 2: 645-52.

8. Perng MD, Cairns L, van den IJssel P, et al. Intermediate filament interactions can be altered by HSP27 and $\alpha B$ crystallin. J Cell Sci 1999; 112: 2099-112.

9. Chavez Zobel AT, Loranger A, Marceau N, et al. Distinct chaperone mechanisms can delay the formation of aggresomes by the myopathy-causing R120G $\alpha$ B-crystallin mutant. Hum Mol Genet 2003; 12: 1609-20. 
10. Wyttenbach A, Sauvageot 0, Carmichael J, et al. Heat shock protein 27 prevents cellular polyglutamine toxicity and suppresses the increase of reactive oxygen species caused by huntingtin. Hum Mol Genet 2002; 11: 1137-51

11. Litt M, Kramer P, LaMorticella DM, et al. Autosomal dominant congenital cataract associated with a missense mutation in the human $\alpha$-crystallin gene CRYAA. Hum Mol Genet 1998; 7: 471-4.
12. Vicart $P$, Caron A, Guicheney $P$, et al. A missense mutation in the $\alpha \mathrm{B}$-crystallin chaperone gene causes a desmin-related myopathy. Nat Genet 1998; 20 . 92-5.

13. Antonellis A, Ellsworth RE, Sambuughin N, et al. Glycyl tRNA synthetase mutations in Charcot-Marie-Tooth disease type 2D and distal spinal muscular atrophy type V. Am J Hum Genet 2003; 72: 1293-9.
14. Hansen JJ, Durr A, Cournu-Rebeix I, et al. Hereditary spastic paraplegia SPG13 is associated with a mutation in the gene encoding the mitochondrial chaperonin Hsp60. Am J Hum Genet 2002; 70 : 1328-32.

15. Benn SC, Perrelet D, Kato AC, et al. Hsp27 upregulation and phosphorylation is required for injured sensory and motor neuron survival. Neuron $2002 ; 36: 45-56$.

\section{NOUVELle}

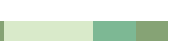

\section{Quand le staphylocoque a besoin de fer, il préfère celui de l'hème

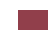

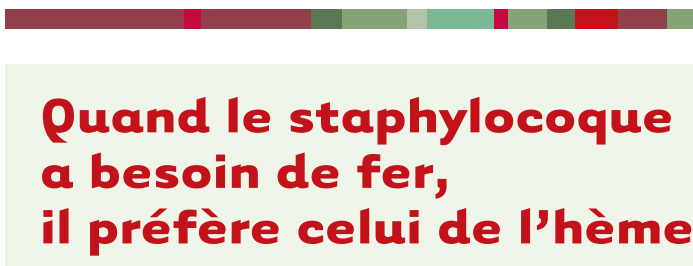

Dominique Labie quasiment tous les organismes vivants, pour des besoins aussi primordiaux que la respiration et la synthèse d'ADN. On explique ainsi la non-prolifération bactérienne dans les profondeurs océaniques par l'absence de fer. La plupart du temps, cependant, la difficulté pour les microorganismes de trouver du fer réside dans le fait que ce dernier est insoluble et/ou inaccessible. Les bactéries, en particulier le staphylocoque, ont développé diverses stratégies de capture du fer. Il peut s'agir de la sécrétion de sidérophores solubles, qui captent le fer externe puis assurent son entrée dans la bactérie par des transporteurs spécifiques. II existe aussi des systèmes d'importation directe de sels de fer. Un premier mécanisme de défense des organismes supérieurs contre l'invasion bactérienne est une limitation drastique du fer libre dans le sang et les tissus. Ce blocage du fer s'effectue majoritairement par sa fixation sur des protéines qui ont pour lui une forte affinité, la lactoferrine et la transferrine. Les sites de fixation du $\mathrm{Fe}^{3+}$ de la transferrine sont, en fait, rarement saturés, laissant toujours un excès de protéine non saturée sous forme d'apotransferrine et assurant ainsi l'élimination virtuelle de tout fer libre.
Un récent travail de chercheurs de I'Université de Chicago (États-Unis) a mis en évidence la stratégie employée par le staphylocoque doré (Staphylococcus aureus) pour se procurer le fer nécessaire à sa croissance et à sa différenciation $[1,2]$. Pour coloniser son hôte, le staphylocoque rencontre un premier obstacle: le manque de fer. La concentration dont il a besoin est de l'ordre de 0,4-4,0 mM, alors que la concentration de fer libre se situe autour de $10^{-9} \mathrm{M}$. Deux sources possibles de capture existent dans l'organisme des mammifères: la transferrine, qui ne représente que $1 \%$ du fer total, et l'hème, qui en contient plus de $80 \%$. Les auteurs ont donc supposé que l'hème devait être utilisé de façon préférentielle. Afin de vérifier cette hypothèse, un marquage spécifique des deux protéines par un isotope stable du fer a été réalisé pour mesurer la consommation de la transferrine $\left[{ }^{57} \mathrm{Fe}\right]$ et de l'hémine $\left[{ }^{54} \mathrm{Fe}\right]$. Les dosages ont été effectués par une spectrométrie de masse en source plasma ICP-MS (inductively coupled plasma-mass spectrometry). Dans une première étape, les bactéries sont placées sur un milieu pauvre en fer jusqu'à une limitation de croissance indicatrice de carence mar-
Département de génétique,

développement et

pathologie moléculaire,

Institut Cochin, 24, rue du

Faubourg Saint Jacques,

75014 Paris, France.

labie@cochin.inserm.fr

tiale, suivie par l'ajout en quantité équimolaire des deux protéines marquées. Les mesures sont ensuite effectuées au bout de 9 heures, 12 heures et en phase stationnaire à 24 heures. Le contenu isotopique des cellules montre, dans les premières heures, un enrichissement ( $x 4$ à 5) en fer héminique en même temps qu'un appauvrissement du milieu. Cette modification des concentrations diminue au cours du temps, mettant en évidence la régulation progressive des autres systèmes de capture (à partir de la transferrine ou de sidérophores). La méthode a aussi permis de préciser la localisation subcellulaire du fer extrait des deux protéines: le fer héminique se fixerait sélectivement à la membrane, celui de la transferrine s'orientant vers le cytoplasme.

Parallèlement à ces mesures isotopiques, une analyse du génome de $S$. aureus a montré sept séquences codant potentiellement pour des transporteurs transmembranaires et présentant une homologie avec des transporteurs connus. Parmi ceux-ci, certains systèmes avaient déjà été mis en évidence par le même groupe de chercheurs. Tout un ensemble de gènes isd $A$ à $F$ (iron-regulated surface determinants) fonctionne comme système d'im- 